\title{
Flat-gain wide-band erbium doped fiber amplifier by combining two difference doped fibers
}

B. A. Hamida

belal@iium.edu.my

S. M. Azooz

A. A. Jasim

T. Eltaif

H. Ahmad

S. Khan

S. W. Harun
Department Electrical and Computer Engineering, International Islamic University Malaysia

Department of Electrical Engineering, Faculty of Engineering, University of Malaya

Department of Electrical Engineering, Faculty of Engineering, University of Malaya

Faculty of Engineering \& Technology, Multimedia University, Melaka, Malaysia

Photonics Research Centre, University of Malaya 50603 Kuala Lumpur, Malaysia

Department Electrical and Computer Engineering, International Islamic University Malaysia

A new erbium-doped fibre amplifier (EDFA) is demonstrated using a combination of newly developed Erbium Zirconia co-doped fiber (Zr-EDF) and the commercial silica-based Erbium-doped fiber (Si-EDF) as the gain medium. Both fibers have a very high concentration of erbium ion. A compact amplifier operating in C-band region is firstly reported using a double-pass configuration. It is shown that average gains of the proposed Zr-EDF amplifier are obtained at approximately $18 \mathrm{~dB}$ with a gain variation of $\pm 2 \mathrm{~dB}$ within C-band region. A flat-gain and wide band operation is achieved by configuring the amplifier in two stages comprising a $2 \mathrm{~m}$ long Zr-EDF and $9 \mathrm{~m}$ long Si-EDF optimised for C- and L-band operations, respectively, in a double-pass parallel configuration. A chirp fibre Bragg grating (CFBC) is used in both stages to ensure double propagation of the signal and thus to increase the attainable gain in both $C$ - and L-band regions. At an input signal power of $0 \mathrm{dBm}$, a flat gain of $15 \mathrm{~dB}$ is achieved with a gain variation of less than $0.5 \mathrm{~dB}$ within a wide wavelength range from 1530 to $1605 \mathrm{~nm}$. The corresponding noise figure varies from 6.2 to $10.8 \mathrm{~dB}$ within this wavelength region.

[DOI: http://dx.doi.org/10.2971/jeos.2015.15015]

Keywords: Double-pass amplifier, wideband amplifier, zirconia-based erbium-doped fiber

\section{INTRODUCTION}

The tremendous growth of the internet and data traffic has created an ever-increasing demand for transmission bandwidth in optical communication systems [1]. The transmission bandwidth can be increased by fully exploiting the low-loss band region of silica fibers using a wide-band amplifier [2, 3]. The wide-band amplification is usually achieved using a hybrid amplifier that combines several amplifiers with different gain bandwidths $[4,5]$. Normally, the wide-band amplifier is constructed by cascading two amplifiers, where the input signal is first de-multiplexed into different bands by the wavelength division multiplexing (WDM) coupler, amplified by amplifiers that are suitable for the corresponding wavelength band, and finally multiplexed again with another WDM coupler. This type of hybrid amplifier has the advantage of extensibility, in which one amplifier can initially work independently while another amplifier can be added into the system according to the demand for expansion.

Up to date, various designs of erbium-doped fiber amplifiers (EDFAs) have been proposed and demonstrated to en- hance the gain, increase the pumping efficiency and reduce the noise figure [6,7]. Many schemes have also been proposed to improve the gain flatness and gain clamping characteristics of the EDFA, which are important to increase the transmission distance in dense wavelength division multiplexing (DWDM) system [8]-[11]. Most recently, few modes EDFAs have been proposed for application in future optical fiber networks based on space-division multiplexing [12].

Extensive research has also been done on a new erbium-doped fiber amplifiers (EDFAs) using various host and co-dopant materials such as silica, alumina, telluride, phosphate, bismuth and others to improve the gain performance, compactness, gain bandwidth, and cost of the devices [13, 14]. The general comparison shows that these materials demonstrate different qualities that have a significant impact on the overall performance and applicability of an optical amplifier. For instance, bismuth co-doped erbium-doped fiber (EDF) has a wider emission bandwidth than silica fibers and allows higher erbium concentrations before detrimental effects such as con- 


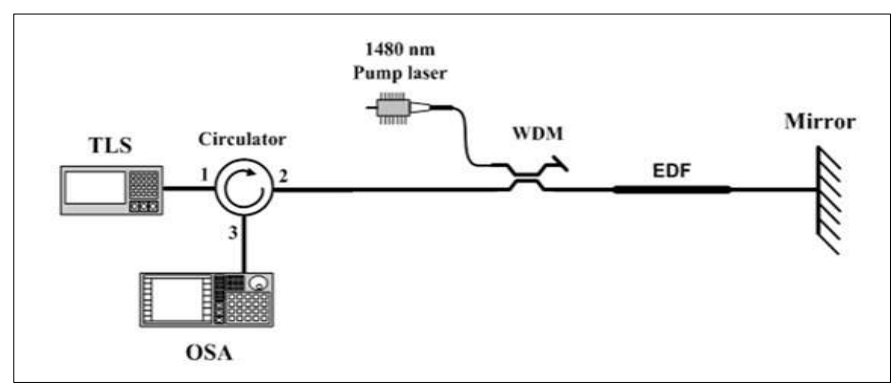

FIG. 1 Proposed double-pass Zr-EDFA configuration.

centration quenching and cluster formation occur, which can be translated to equal gain in a shorter fiber length. In the choice of glass host, many researchers have chosen to use high silica glass due to its proven reliability and compatibility with conventional fiber-optic components. In this paper, a compact double-pass optical amplifier is proposed for improving the attainable gain in both newly developed zirconia-co-doped and the conventional silica-based EDFAs. A cascaded amplifier in both parallel and series configuration is also proposed for flat-gain and wide-band operation.

\subsection{Double-pass compact optical amplifiers}

Figure 1 shows the single-stage EDFA in double-pass setup, which utilizes a highly doped EDF as a gain medium and a broadband fiber mirror as a reflector. The performance of the amplifier is compared for two different gain media; a silicabased commercial EDF and a newly developed Zr-based EDF (Zr-EDF). The silica-based EDF has an erbium ion concentration of $2200 \mathrm{ppm}$ with an optimised length of $73 \mathrm{~cm}$. The $\mathrm{Zr}$ based EDF used is obtained from a fiber preform, which was fabricated in a ternary glass host, zirconia-yttria-aluminum co-doped silica fiber using a modified chemical vapor deposition (MCVD) process [15]. The erbium ion is doped into a zirconia yttria-aluminosilicate based glass using a solution doping process. With a combination of both $\mathrm{Zr}$ and $\mathrm{Al}$, we could achieve a high erbium doping concentration in the glass host without any phase separations of rare-earths. Only a minor amount of $\mathrm{Y}_{2} \mathrm{O}_{3}$ is used in the preform to prevent cracking problem. A fiber of $125 \mu \mathrm{m}$ in diameter is drawn from the fabricated preform at temperature of around $2000^{\circ} \mathrm{C}$ using the conventional fiber drawing technique. The peak absorption of the $\mathrm{Zr}$ fiber at $978 \mathrm{~nm}$ was measured to be $14.5 \mathrm{~dB} / \mathrm{m}$, which is equivalent to the erbium ions concentration of $2800 \mathrm{ppm} w \mathrm{w}$. The fiber length is fixed at $57 \mathrm{~cm}$ in the experiment so that the total amount of erbium ions in both tested fibers is the same.

\section{EXPERIMENTAL SETUP}

In the experiment, the EDF is forward pumped by $1480 \mathrm{~nm}$ laser diode via 1480/1550 nm WDM coupler. The $1480 \mathrm{~nm}$ pump power is fixed at the maximum power of $150 \mathrm{~mW}$. A mirror is located at the other end of the EDF to reflect the signals back to the gain medium and allow double propagation. An optical circulator is used to extract the twice amplified output signal. A tunable laser source (TLS) is used in conjunction with an optical spectrum analyzer (OSA) to characterize the double-pass EDFA. The performance of the double-pass

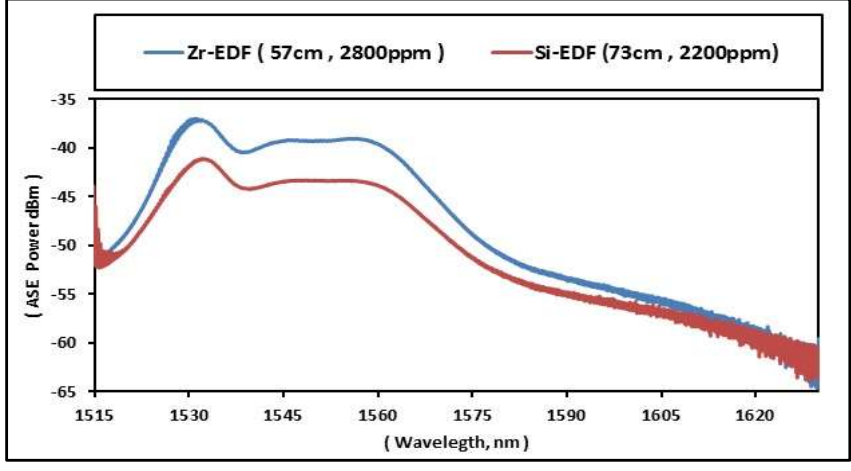

FIG. 2 ASE spectra from Zr-EDF and Si-EDF under the same pumping power.

EDFA is also compared to that of the single-pass one, which is obtained by removing the mirror and measuring the amplified signal at the output end of the EDF.

Figure 2 compares the amplified spontaneous emission (ASE) spectrum of the proposed double-pass EDFA with the conventional EDFA configured with a silica-based EDF (Si-EDF). It is shown that the Zr-EDFA gives an average power of $5 \mathrm{~dB}$ higher than its Si-based (I-25) EDFA counterpart at C-band region. The Zr-EDFA uses only $57 \mathrm{~cm}$ gain medium with the same pumped power of equivalent length leading to a considerably reduced signal losses.

The gain and noise figure are measured over a wide wavelength region ranging from 1520 to $1620 \mathrm{~nm}$ for both amplifiers. Figure 3 shows the result when the pump and input signal powers is fixed at $150 \mathrm{~mW}$ and $-30 \mathrm{dBm}$, respectively. It is obtained that the gain of $\mathrm{Zr}$ co-doped EDFA is higher than that of Si-based amplifier especially at $1560 \mathrm{~nm}$ region. This is attributed to the population inversion which is higher in the $\mathrm{Zr}$-EDF, which has a shorter length. The gain improvement of more than $5 \mathrm{~dB}$ is obtained at wavelength region ranging from 1530 to $1565 \mathrm{~nm}$ due to the efficient use of pump power with a shorter gain medium. The average gains of the proposed Zr-EDFA are obtained at approximately $18 \mathrm{~dB}$ with a gain variation of $\pm 2 \mathrm{~dB}$ within this region. The gain spectrum also broadens due to the enhancement of the inhomogeneous distribution of erbium ion sites and the suppression of signal excited state absorption (ESA). Figure 3 also shows that the noise figure of Zr-EDFA remains within the acceptable limits of $+8 \mathrm{~dB}$ all the way over the entire wavelength region. However, the noise figure of the Si-based EDF goes higher even to reach $+18 \mathrm{~dB}$ at $1620 \mathrm{~nm}$. The higher noise figure of SiEDFA is due to inefficient population inversion especially at the input part of the Zr-EDF due to the high erbium concentration. The noise figures are obtained at an average of $10 \mathrm{~dB}$ within $1520 \mathrm{~nm}$ to $1580 \mathrm{~nm}$ for both double-pass EDFAs. The noise figure is expected to reduce if we use a longer EDF as the gain medium. The optimization to reduce the component and splicing loss may also reduce the noise figure.

\subsection{Double-pass compact wide-band optical amplifiers}

Figure 4(a) shows the proposed wideband EDFA employing a piece of Zr-EDF and Si-EDF for C-band and L-band opera- 


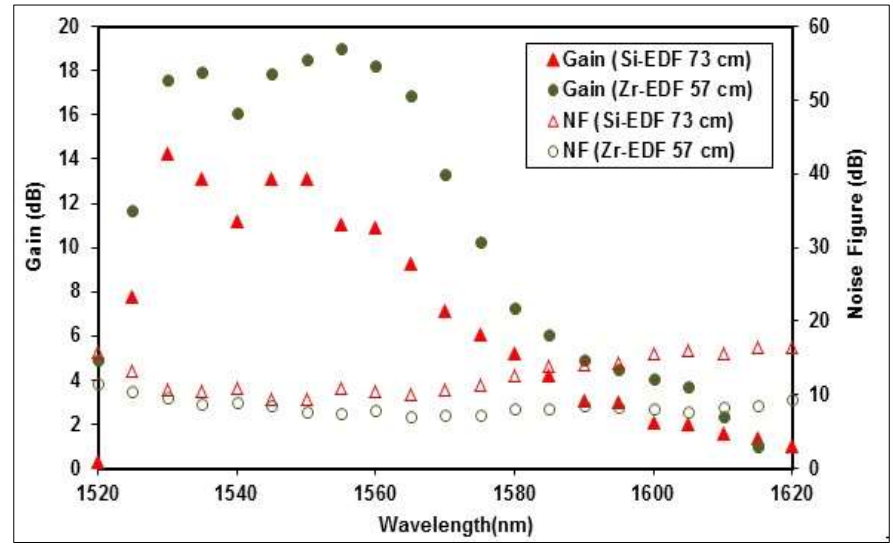

FIG. 3 Gain and NF of Zr-EDFA and Si-EDFA at input signal of $-30 \mathrm{dBm}$.

tion respectively, in double-pass parallel configuration. At the input of the optical amplifier, a C/L-band WDM coupler is used to separate the WDM signals to C- and L-bands. The C-band and L-band signals are amplified by a piece of $2 \mathrm{~m}$ and $9 \mathrm{~m}$ long Zr-EDF and Si-EDF, which are forward pumped by a $980 \mathrm{~nm}$ and $1480 \mathrm{~nm}$ laser diode, respectively. The $980 \mathrm{~nm}$ and $1480 \mathrm{~nm}$ pumps are marked as P1 and P2 to help us in the discussion. At the end of each stage, a broadband CFBG operating at C-or L-band is incorporated to reflect the C-or L-band signals for double-pass operation. These signals are combined together by the C/L-band WDM coupler at the input port of the amplifier before they are routed to the output port via optical circulator as shown in Figure 4(a). The insertion loss of the WDM couplers is assumed to be 0.9 and $1.8 \mathrm{~dB}$ in the Cand L-band region, respectively.

The performance of the hybrid amplifier with serial configuration of Figure 4(b) is also investigated for comparison purpose. The serial amplifier uses similar components as those of the proposed parallel amplifier of Figure 4(a). The C-band CFBG is placed in between the two stages to act as a reflector for the C-band EDFA. It reflects C-band signal for doublepass operation and allows the L-band signal to be transmitted so that it can be amplified by the second stage of the amplifier. The amplified signal is then reflected back into the system by the L-band CFBG. Both CFBGs used in the experiment are commercially obtained. The C-band CFBG has a reflectivity of more than $90 \%$ centred at the wavelength of $1545 \mathrm{~nm}$ with a bandwidth of about 40nm while the L-band CFBG has a reflectivity of more than $98 \%$ centred at $1592 \mathrm{~nm}$ with a bandwidth of about $50 \mathrm{~nm}$. In this experiment, the gain and noise figures of both EDFAs are characterized using a TLS used in conjunction with an OSA.

Figure 5 compares the measured gain and noise figure spectrum characteristics between the proposed parallel and serial double-pass amplifiers. In the experiment, P1 and P2 powers are fixed at 112 and $150 \mathrm{~mW}$ while input signal power is fixed at $0 \mathrm{dBm}$. As shown in the figure, wide-band operation is achieved in both amplifiers, which are operating in wavelength region from $1525 \mathrm{~nm}$ to $1615 \mathrm{~nm}$. The gain of the parallel amplifier is maintained at $15 \mathrm{~dB}$ within a wavelength region from $1530 \mathrm{~nm}$ to $1605 \mathrm{~nm}$ with a gain variation of less than $0.5 \mathrm{~dB}$. On the other hand, the serial amplifier shows a lower gain, which varies from 9 to $14.6 \mathrm{~dB}$ in the

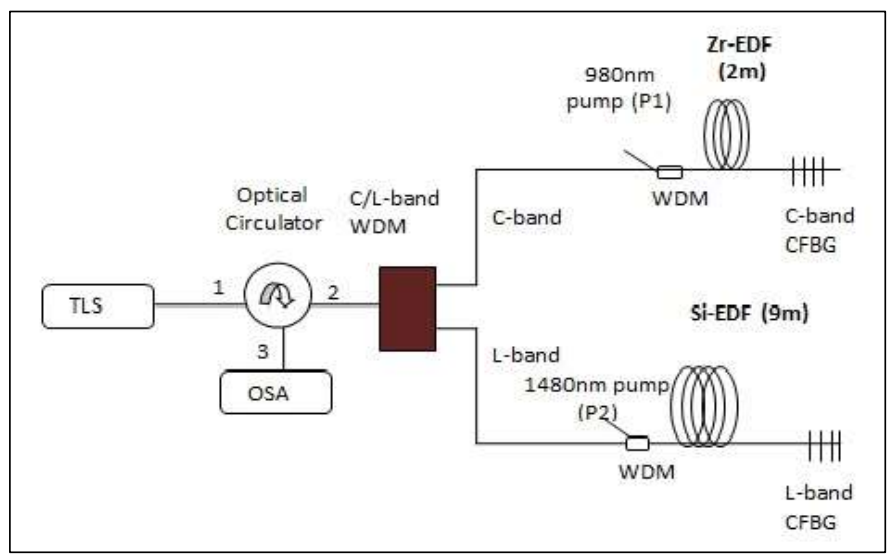

(a) Parallel

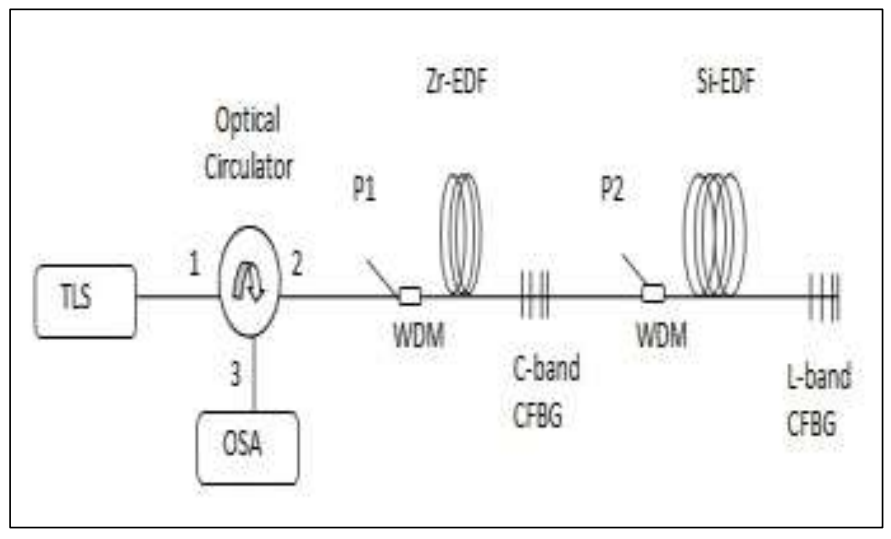

(b) Serial

FIC. 4 Configuration of two-stage double-pass EDFAs in (a) parallel and (b) serial configurations.

C-band region while showing a slightly higher gain within 10 to $17.6 \mathrm{~dB}$ in the L-band region as compared to that of the parallel one. In the serial amplifier of Figure 4(b), the reflected forward L-band ASE passes through the C-band CFBG and is amplified by the first stage amplifier, which in turn suppresses the amplifier's gain in the C-band region. Furthermore, at $1570 \mathrm{~nm}$, the gain for the serial configuration suddenly increases due to the shift in the amplification medium length from $2 \mathrm{~m}$ to $9 \mathrm{~m}$. On the other hand, the noise figure of the parallel amplifier varies from 7.4 to $11.2 \mathrm{~dB}$ within the wavelength region of $1530 \mathrm{~nm}$ to $1615 \mathrm{~nm}$. These values are relatively smaller compared to that of the serial amplifier except for wavelength of $1565 \mathrm{~nm}$. At around $1565 \mathrm{~nm}$, the insertion loss of C/L-band WDM is the highest and therefore the noise figure is relatively higher at this region. Compared to the parallel amplifier, the L-band noise figure in the serial amplifier is higher due to the backward ASE from the second stage, which was reflected back to the gain medium and thus reduces the population inversion at the input part of the L-band EDFA. Compared to the conventional EDFA, the noise figure of the both amplifiers is slightly higher due to the implementation of double-pass scheme.

The $980 \mathrm{~nm}$ pump power or P1 which controls the C-band gain is varied to improve the flatness of the gain spectrum. Figure 6(a) and 6(b) show the gain and noise figure spectra for the parallel amplifier at various $\mathrm{P} 1$ powers for input signal powers of $-30 \mathrm{dBm}$ and $0 \mathrm{dBm}$, respectively. As shown in Fig- 


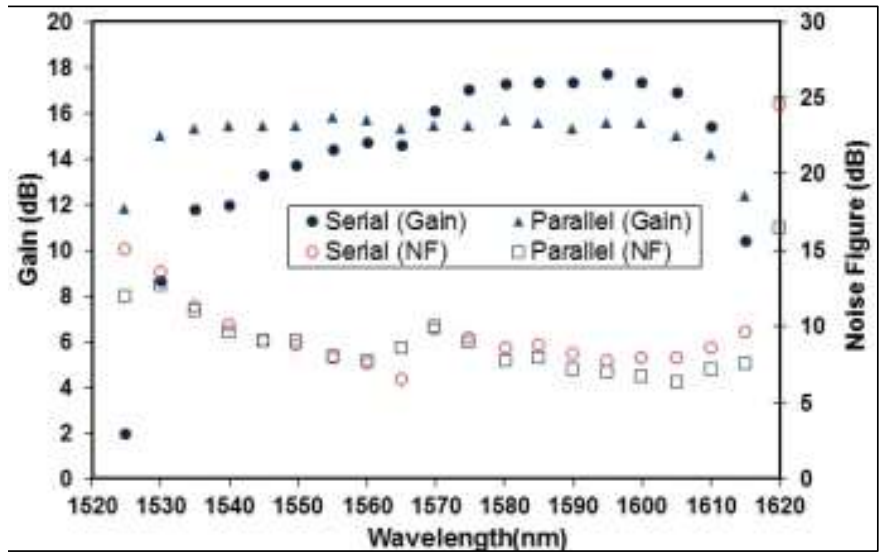

FIG. 5 Comparison of gain and noise figure - blank dotted plots show noise figure and shaded dotted plots show gain for serial and parallel configuration.

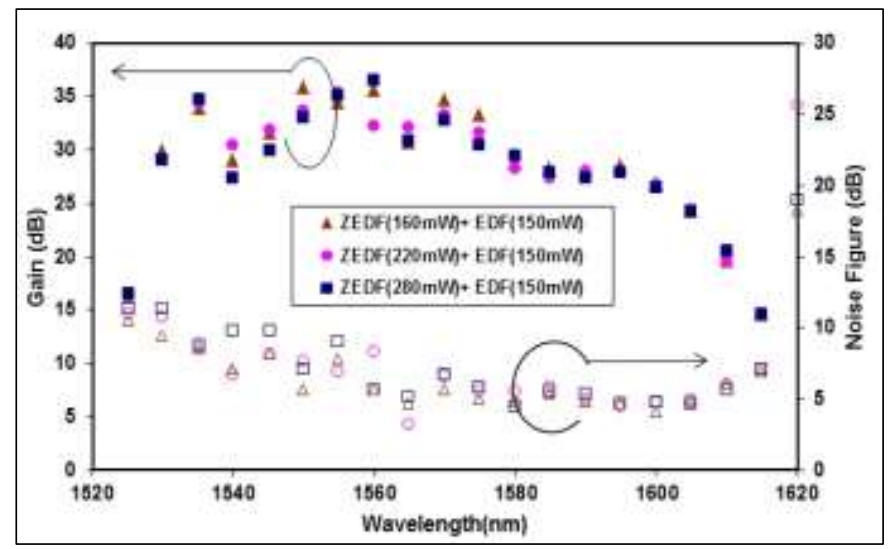

(a) Input signal power of $-30 \mathrm{dBm}$

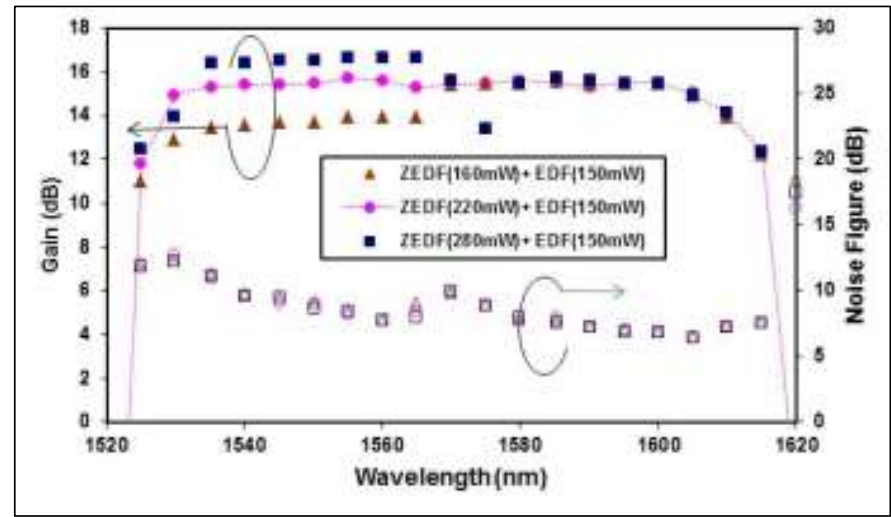

(b) Input signal power of $0 \mathrm{dBm}$

FIG. 6 Gain and noise figure spectra of the amplifier configured in parallel setup at different pump power combinations for two different input signal powers of (a) $30 \mathrm{dBm}$ and (b) $0 \mathrm{dBm}$.

ure 6(a) the C-band small signal gain $(-30 \mathrm{dBm})$ fluctuates at around $32 \mathrm{~dB}$ as the $\mathrm{P} 1$ pump power varies from $160 \mathrm{~mW}$ to $280 \mathrm{~mW}$. For instance, at input signal wavelength of $1550 \mathrm{~nm}$, the gain decreases from $36.5 \mathrm{~dB}$ to $33 \mathrm{~dB}$ as the pump power changes from $160 \mathrm{~mW}$ to $280 \mathrm{~mW}$. The signal variation at small input signal power is most likely due to the gain saturation effect. Some of the C-band photons are absorbed by the erbium ions to emit photons operating at L-band region. These L-band photons pass through the CFBG and diminishes. Therefore, the L-band small signal gain and noise figure spectra are al- most unchanged as shown in Figure 6(a). On the other hand, at input signal power of $0 \mathrm{dBm}$, the $\mathrm{C}$-band gain increases as the P1 pump power increases from $160 \mathrm{~mW}$ to $280 \mathrm{~mW}$ as shown in Figure 6(b). The optimum pump power for flat gain operation is observed to be around $220 \mathrm{~mW}$. The noise figure spectrum is seen to be unaffected by the change of pump power of the C-band amplifier.

\section{CONCLUSION}

A compact double-pass Zr-EDFA and Si-EDFA operating in $\mathrm{C}$-band region is demonstrated using a $57 \mathrm{~cm}$ long $\mathrm{Zr}-\mathrm{EDF}$ and a $73 \mathrm{~cm}$ long Si-EDF, respectively as a gain medium. It is found that the Zr-EDFA is more efficient than the Si-EDFA. It has an average gain of $18 \mathrm{~dB}$ with a gain variation of $\pm 2 \mathrm{~dB}$ at C-band region. By cascading both double-pass amplifiers in parallel and series, a wide-band operation covering both $C$ and L-band region was realized. In the experiment, a CFBG is used in both stages to enable double-pass operation. A flatgain and wide band operation is achieved by configuring the amplifier in two stages comprising a $2 \mathrm{~m}$ long Zr-EDF and $9 \mathrm{~m}$ long Si-EDF optimised for C- and L-band operations, respectively. For instance, a flat gain of $15 \mathrm{~dB}$ is achieved at an input signal power of $0 \mathrm{dBm}$ with a gain variation of less than $0.5 \mathrm{~dB}$ within a wide wavelength range from 1530 to $1605 \mathrm{~nm}$. The corresponding noise figure varies from 6.2 to $10.8 \mathrm{~dB}$ within this wavelength region.

\section{ACKNOWLEDGEMENTS}

Financial support for this work by MOSTI (SF014-2014) and UM (RP008C-13AET) is gratefully acknowledged.

\section{References}

[1] A. E. A. Mohammed, and A. N. Z. Rashed, "Ultra wide band (UWB) of optical fiber Raman amplifiers in advanced optical communication networks," J. Media Commun. Stud. 1, 56-72 (2009).

[2] S. W. Harun, N. Tamchek, P. Poopalan, and H. Ahmad, "Doublepass L-band EDFA with enhanced noise figure characteristics," IEEE Photon. Technol. Lett. 15, 1055-1057 (2003).

[3] S. W. Harun, N. K. Saat, and H. Ahmad, "An efficient S-band erbiumdoped fiber amplifier using double-pass configuration," IEICE Electron. Expr. 2, 182-185 (2005).

[4] X. Wang, Q. Nie, T. Xu, X. Shen, S. Dai, and N. Gai, “Tm³ -doped tellurite glass with $\mathrm{Yb}^{3+}$ energy sensitized for broadband amplifier at 1400-1700 nm bands," J. Rare Earth. 26, 907-911 (2008).

[5] A. J. G. Ellison, J. E. Dickinson, D. E. Goforth, D. L. Harris, J. T. Kohli, J. D. Minelly, B. N. Samson, et al., "Hybrid erbium silicate conventional-band fiber amplifier with ultra-low gain ripple," in Proceedings to optical Amplifiers and their Applications, NW2 (Optical Society of America, Nara, 1999).

[6] P. F. Wysocki, N. Park, and D. DiGiovanni, "Dual-stage erbiumdoped, erbium/ytterbium-codoped fiber amplifier with up to +26 dBm output power and a $17 \mathrm{~nm}$ flat spectrum," Opt. Lett. 21, 1744-1746 (1996). 
[7] E. Desurvire, D. Bayart, B. Desthieux, and S. Bigo, Erbium-doped fiber amplifiers, device and system developments (John Wiley $\mathrm{A}$ Sons Inc., New York, 2002).

[8] M. Kakui, T. Kashiwada, K. Nakazato, M. Onishi, C. Fukuda, M. Shigematsu, and M. Nishimura, "Design optimization of hybrid erbium-doped fiber amplifiers for WDM transmission systems," Opt. Fiber Technol. 3, 123-133 (1997).

[9] J. Nilsson, and B. Jaskorzynska, "Alternating cascade of spectrally different erbium-doped fiber amplifiers for link-loss-insensitive long-haul WDM transmission," J. Lightwave Technol. 17, 434-444 (1999).

[10] A. Bjarklev, "Hybrid erbium-doped fiber for gain flattened operation," Opt. Fiber Technol. 3, 72-76 (1997).

[11] S. W. Harun, and H. Ahmad, "Gain clamped two-stage doublepass L-band EDFA with a single fibre Bragg grating," Chinese Phys. Lett. 21, 1954-1957 (2004).
[12] L. Bigot, G. Le Cocq, and Y. Quiquempois, "Few-mode erbiumdoped fiber amplifiers: a review," J. Lightwave Technol. (2015) article in press.

[13] X. S. Cheng, R. Parvizi, H. Ahmad, and S. W. Harun, "Wide-band bismuth-based erbium-doped fiber amplifier with a flat-gain characteristic," IEEE Photon. J. 1, 259-264 (2009).

[14] R. Schermer, W. Berglund, C. Ford, R. Ramberg, and A. Gopinath, "Optical amplification at $1534 \mathrm{~nm}$ in erbium-doped zirconia waveguides," IEEE J. Quantum Elect. 39, 154-159 (2003).

[15] H. Ahmad, K. Thambiratnam, M. C. Paul, A. Z. Zulkifli, Z. A. Chani, and S. W. Harun, "Fabrication and application of zirconia-erbium doped fibers," Opt. Mater. Express 2, 1690-1701 (2012). 